\title{
Gas Chromatography Detection Protocol of Short-chain Fatty Acids in Mice Feces
}

Chaozheng Zhang*, An Liu, Tianshuang Zhang, Yi Li and Hua Zhao*

Key Laboratory of Ministry of Education Industrial Fermentation Microbiology, Tianjin Key Laboratory of Industrial Microbiology, Tianjin Engineering Research Center of Microbial Metabolism and Fermentation Process Control, College of Biotechnology, Tianjin University of Science and Technology, Tianjin, 300457, P. R. China

*For correspondence: zhaohua@tust.edu.cn; zhangchaozheng@tust.edu.cn

[Abstract] Short-chain fatty acids (SCFAs), which are formed mainly by bacteria fermenting undigested carbohydrates in the colon, they are based on the number of carbon atoms in the carbon chain. Organic fatty acids with less than 6 carbon atoms are called short-chain fatty acids. SCFAs are closely related to various aspects of the human body, so more and more researchers concentrate on SCFAs. This protocol describes, a direct injection gas chromatography detection method with a pretreatment method for extracting SCFA from mice feces by combining acidification. The corresponding sample limit of quantization (LOQ) and limit of detection (LOD) are $0.8-1.0 \mathrm{mg} / \mathrm{L}$ and $0.5-0.8 \mathrm{mg} / \mathrm{L}$, respectively. The correlation coefficient of calibration curve is greater than 0.999 . The recovery rate of the spiked standard is $80 \%-102 \%$. This method can be used to analyze and determine SCFAs in mice feces. Therefore, this is an economical, effective and reproducible method for SCFAs measurement in mice samples.

Keywords: Short-chain fatty acids, Acidification, Extraction, Gas chromatography, Biological samples

[Background] Short-chain fatty acids (SCFAs) are important molecules that regulate and control biological functions (Siri-Tarino et al., 2015). SCFAs typically refer to volatile organic fatty acids with less than six carbon atoms (Wong et al., 2006; Marette and Jobin, 2015). They mainly include acetic acid, propionic acid, isobutyric acid, butyric acid, isovaleric acid, and valeric acid. SCFAs are mainly produced by the intestinal microbiota, which ferments undigested carbohydrates and dietary fiber in the colon. (Henningsson et al., 2001). Many studies have shown that branched fatty acids are derived from branched-chain amino acids and leucine (Koh et al., 2016). A small amount of branched-chain fatty acids are generated by fermenting undigested proteins (Cummings and Englyst, 1987), such as isobutyric acid and isovaleric acid. Numerous types of SCFAs are present in the colon, and have different functions, because bacteria are complex and fermentation substrates vary in the colon (Topping and Clifton, 2001). These metabolites are formed and absorbed in the colon and then transported through the hepatic vein to the liver. Only approximately 5\% of SCFAs are excreted in feces and the remaining are absorbed by colon cells (Ruppin et al., 1980; Hu et al., 2010; Stumpff, 2018). SCFAs play an important role in maintaining the normal function of the large intestine and the morphology and function of colonic epithelial cells. Among other features, SCFAs contributes to the main role of the intestinal flora in the homeostasis of the host. Studies have shown that propionic acid, 
butyric acid and 3-hydroxybutyric acid, which are signaling molecules, play important roles in activating $G$ protein-coupled receptors and inhibiting histone deacetylase activity (Johnstone, 2002; Xiong et al., 2004). The most abundant molecules in SCFAs are acetic acid, propionic acid, and butyric acid, which are usually present in deprotonated form. SCFAs are quickly absorbed by the hindgut and store energy. Acetic acid provides $10 \%$ of the body's energy expenditure and is an important component of the collective energy source (Hu et al., 2010). The close relationship between SCFAs content in the human body and human health has always been the focus of research (O'Keefe, 2016). Therefore, a method for the rapid qualitative and quantitative analyses of SCFAs in complex biological matrices is needed to be developed (Fiorini et al., 2015).

Many detection techniques can be used for the qualitative and quantitative identification of SCFAs. Those include high-performance liquid chromatography (HPLC) (Torii et al., 2010; de Sá et al., 2011; Schiffels et al., 2011; De Baere et al., 2013), capillary electrophoresis (Saavedra et al., 2000), and ion exchange HILC (Horspool et al., 1991). However, these methods have several limitations in terms of poor sensitivity and low recovery (Saavedra et al., 2000; Destandau et al., 2005; Horspool et al., 1991). Gas chromatography (GC) is the most commonly used method for SCFA analysis because SCFAs are volatile. SCFA biological samples are prepared differently in terms of sample processing such as pretreatment, distillation, ultrafiltration, and extraction (Zhao et al., 2006; Fiorini et al., 2015; Hoving et al., 2018). However, the tedious and time-consuming pretreatment process may be one of the reasons for the decrease in detection sensitivity and recovery of SCFA.

Therefore, GC was chosen to detect SCFA, but a pretreatment method for processing biological samples needs to be developed. Such method must not only be simple and easy to implement, but also time-efficient. In this study, a simple and effective pretreatment method for the rapid qualitative and quantitative determination of SCFAs in mice feces via GC was developed. This approach can provide a basis for the analysis of SCFAs in other biological samples.

\section{Materials and Reagents}

1. $100-1,000 \mu l$ pipette tip (Biosharp, catalog number: BS-1000-T)

2. Micro-centrifuge tube, $5 \mathrm{ml}$ (Biosharp, catalog number: BS-50-M)

3. Disposable sterile syringe, $1 \mathrm{ml}$ (Zhengzhou Kangjia Medical Equipment Co., Ltd)

4. Clear short thread wide mouth sample bottle, $2 \mathrm{ml}$, vial with patch $\left(32^{*} 11.6 \mathrm{~mm}\right.$ ) (Agilent Technologies, catalog number: 5182-0715)

5. Blue screw cap (9 mm) (Agilent Technologies, catalog number: 5182-0717)

6. Disposable sterile needle filter, Nylon 66, Aperture: $0.22 \mu \mathrm{m}$ (Tianjin cypress Experimental Equipment Co., Ltd, catalog number: SFNF1322)

7. Mice feces samples, stored at $-20{ }^{\circ} \mathrm{C}$ after collection

8. Hydrochloric acid, $37 \%$ (v/v) (Tianjin Fengchuan Chemical Reagent Technology Co., Ltd. CAS: 7647-01-0) 
9. Acetic acid, standard for GC, $\geq 99.8 \%$ (GC) (Aladdin Industrial Corporation, catalog number: A116165)

10. Propionic acid, standard for GC, $\geq 99.5 \%$ (GC) (Aladdin Industrial Corporation, catalog number: P110443)

11. Butyric acid, Standard for GC, $>99.5 \%$ (GC) (Aladdin Industrial Corporation, catalog number: B110439)

12. Isobutyric acid, Standard for GC, $\geq 99.5 \%$ (GC) (Aladdin Industrial Corporation, catalog number: I103521)

13. Valeric acid, Standard for GC, $\geq 99.5 \%$ (GC) (Aladdin Industrial Corporation, catalog number: V108269)

14. Isovaleric acid, Standard for GC, $\geq 99.5 \%$ (GC) (Aladdin Industrial Corporation, catalog number: 1108280)

15. Ethyl acetate, Analytical purity, $\geq 99.5 \%$ (Tianjin Fuchen Chemical Reagent Co., Ltd.)

16. $1 \mathrm{M} \mathrm{HCl}$ solution (see Recipes)

17. SCFAs single standard solution (see Recipes)

18. SCFAs mixed standard solution (see Recipes)

19. Dilute SCFAs single standard solution with a dilution factor of 2, 4, 8, 10 times (see Recipes)

20. SCFAs mixed standard solution (see Recipes)

21. Dilute SCFAs mixed standard solution with 2, 4, 8, 10 Dilution Times (see Recipes)

\section{Equipment}

1. $50 \mathrm{ml}$ volumetric flask

2. Beaker

3. $100-1,000 \mu l$ pipette gun (Eppendorf, model: research plus)

4. A gas chromatograph (Agilent 7890A, USA) equipped with flame ionization detector and an automatic sampler (Autosampler, model: 7683B)

5. DB-FFAP capillary column with polyethylene glycol modified by terephthalic acid as stationary phase (30 m x $320 \mu \mathrm{m} \times 0.5 \mu \mathrm{m}$ ) (Agilent Technologies, USA, catalog number: 123-3233)

6. Stainless steel spoon, $180 \mathrm{~mm}$ long, with the widest diameter of $3 \mathrm{~mm}$

7. Analytical balance (METTLER TOLEDO Instruments (Shanghai) Co., Ltd., model: ME204/02)

8. Vortex mixer (Aoran Science and Technology Co., Ltd., model: SI-0246-G560E)

9. Centrifuge (Changsha High-Tech Industrial Development Zone Xiangyi Centrifuge Instrument Co., Ltd., model: H1650R)

10. Ultrasonic cleaner (Kunshan Hechuang Ultrasonic Instrument Co., Ltd., model: KH-500E)

\section{Software}

1. Agilent ChemStation was used to analyze data (B.0403) 


\section{Procedure}

A. Sample collection

1. Select mice in similar growth status at 6-8 weeks.

2. Collect fecal samples from 12 mice, approximately $300 \mathrm{mg}$ per sample (stored at $-20^{\circ} \mathrm{C}$ ). Used for the determination of SCFAs and recovery.

3. Mix 12 samples fully, weigh about $100 \mathrm{mg}$ of the mixed sample and place it in a $5 \mathrm{ml}$ centrifuge tube. Repeat the weighing of the 22 samples. Use three of them to test SCFAs in parallel, and use the remaining 19 to determine the rate of recovery. Number and record the sample quality (stored at $-20^{\circ} \mathrm{C}$ ).

B. Sample pretreatment

1. Keep all biological samples frozen at $-20^{\circ} \mathrm{C}$ until the day of analysis.

2. Add $3 \mathrm{ml} \mathrm{HCl} 1 \mathrm{M}$ to each sample, vortex for $1 \mathrm{~min}$, mix thoroughly until no obvious clumping.

3. Place the well-mixed centrifuge tube in an ice-water bath in the beaker, and conduct ultrasonic extraction for $10 \mathrm{~min}$ (see Video 1). Set the temperature to room temperature and the frequency is $40 \mathrm{k} \mathrm{Hz}$.

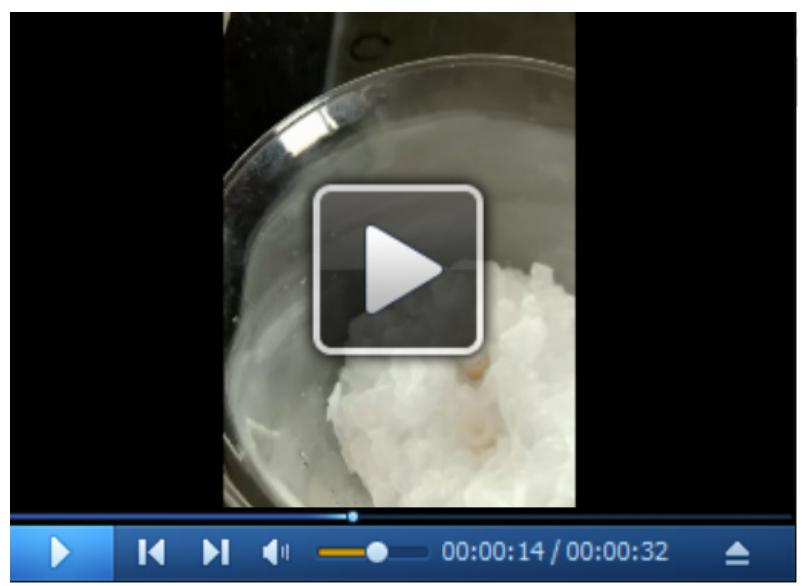

Video 1. Ice water bath

4. Centrifuge the sample at $4{ }^{\circ} \mathrm{C}, 9,710 \times g$ for $15 \mathrm{~min}$.

5. Use a pipette to transfer $1 \mathrm{ml}$ of supernatant into a $5 \mathrm{ml}$ centrifuge tube (see Figure 1). 


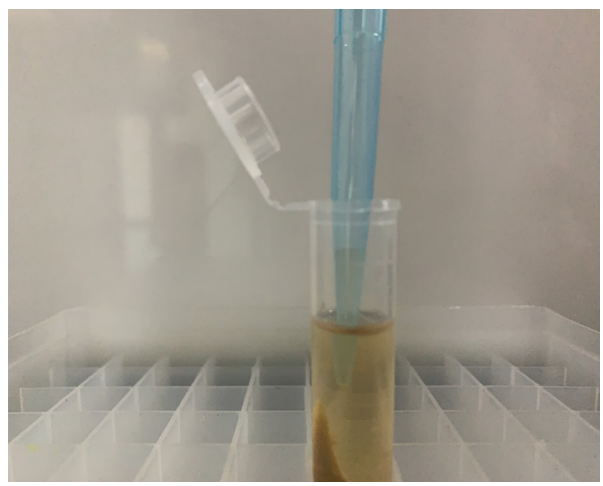

Figure 1. Absorb the supernatant

6. Add $1 \mathrm{ml}$ of ethyl acetate to the supernatant, invert the centrifuge tube to completely mix the supernatant (see Video 2).

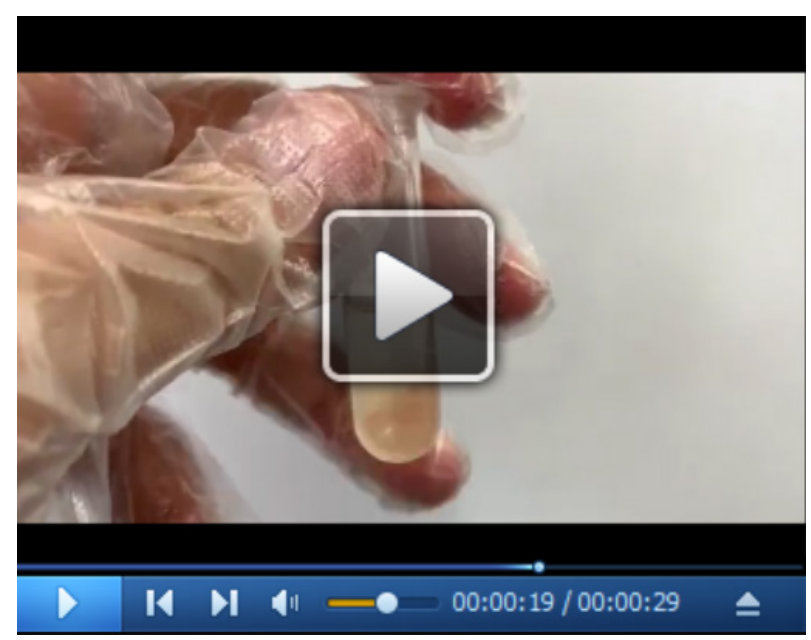

Video 2. Extraction

7. Centrifuge the sample at $4{ }^{\circ} \mathrm{C}, 9,710 \times g$ for $15 \mathrm{~min}$.

8. Aspirate the supernatant with disposable sterile syringe and filter through a $0.22 \mu \mathrm{m}$ organic filter membrane. Transfer the filtered supernatant to Clear short thread wide mouth sample bottle, and inject into GC for analysis (If the sample stored in the bottle cannot be analyzed as soon as possible, it can be stored at $-20^{\circ} \mathrm{C}$ for $1-2$ days) (see Figure 2). 


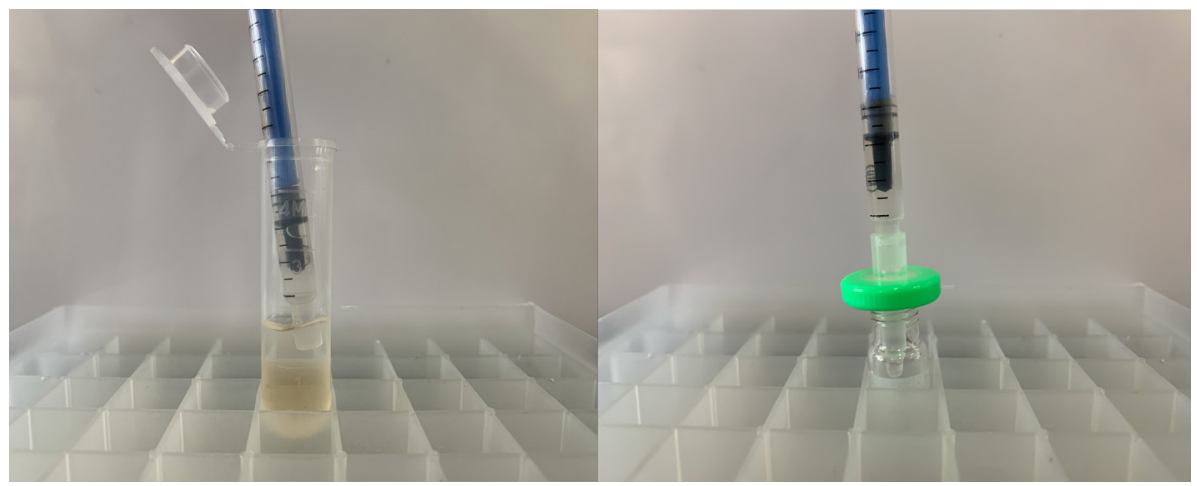

Figure 2. Aspirate the supernatant and filter

C. Gas Chromatography (GC) experimental conditions

Set the GC program according to conditions mentioned in Table 1.

Table 1. GC experimental conditions

\begin{tabular}{ll}
\hline GC program & Condition setting \\
\hline Chromatographic column & DB-FFAP(See equipment for details) \\
Inlet temperature & $250{ }^{\circ} \mathrm{C}$ \\
Injection volume & $1 \mu \mathrm{ll}$ \\
Shunting ratio & $1: 1$ \\
Carrier gas type & $\mathrm{N} 2$ \\
Carrier gas velocity & $2 \mathrm{ml} / \mathrm{min}$ \\
& Adjust the temperature program of the oven to $90{ }^{\circ} \mathrm{C}$, maintain \\
Oven temperature conditions & for $5 \mathrm{~min}$ and increase to $200{ }^{\circ} \mathrm{C}$ at a rate of $20{ }^{\circ} \mathrm{C} /$ min and \\
& maintain for 3 min. \\
FID detector & $250{ }^{\circ} \mathrm{C}$ \\
Flow of the H2 & $40 \mathrm{ml} /$ min \\
Flow of the synthetic air & $450 \mathrm{ml} /$ min \\
Sample probe cleaning & ethyl acetate \\
\hline
\end{tabular}

D. Standard preparation and accuracy analysis

1. Prepare SCFAs single standard solution: using ethyl acetate as solvent, the maximum concentrations of acetic acid, propionic acid, isobutyric acid, butyric acid, isovaleric acid and valeric acid were adjusted to 2, 0.5, 0.1, 0.5, 0.5 and $0.1 \mathrm{~g} / \mathrm{L}$, respectively (see Recipe 2 for specific operation).

2. Dilute the mixed solution of the maximum concentration with ethyl acetate 2, 4, 8 and 10 times (see Recipe 3 for specific operation).

3. Prepare SCFAs mixed standard solution: using ethyl acetate as solvent, the maximum concentrations of acetic acid, propionic acid, isobutyric acid, butyric acid, isovaleric acid and 
valeric acid in the mixed solution were adjusted to $2,0.5,0.1,0.5,0.5$ and $0.1 \mathrm{~g} / \mathrm{L}$, respectively (see Recipe 4 for specific operation).

4. Dilute the mixed solution of the maximum concentration with ethyl acetate 2, 4, 8 and 10 times (see Recipe 5 for specific operation).

5. SCFAs were injected into GC in accordance with the concentration gradient (Table 2) to determine the calibration curve of the SCFAs.

Table 2. Standard Solution Concentration of 6 SCFAs

\begin{tabular}{lllllll}
\hline Level & $\begin{array}{l}\text { Acetic } \\
(\mathrm{g} / \mathrm{L})\end{array}$ & $\begin{array}{l}\text { Propionic } \\
(\mathrm{g} / \mathrm{L})\end{array}$ & $\begin{array}{l}\text { i-Butyric } \\
(\mathrm{g} / \mathrm{L})\end{array}$ & $\begin{array}{l}\text { Butyric } \\
(\mathrm{g} / \mathrm{L})\end{array}$ & $\begin{array}{l}\text { i-Valeric } \\
(\mathrm{g} / \mathrm{L})\end{array}$ & $\begin{array}{l}\text { Valeric } \\
(\mathrm{g} / \mathrm{L})\end{array}$ \\
\hline 1 & 2 & 0.5 & 0.1 & 0.5 & 0.5 & 0.1 \\
2 & 1 & 0.25 & 0.05 & 0.25 & 0.25 & 0.05 \\
3 & 0.5 & 0.125 & 0.025 & 0.125 & 0.125 & 0.025 \\
4 & 0.25 & 0.0625 & 0.0125 & 0.0625 & 0.0625 & 0.0125 \\
5 & 0.2 & 0.05 & 0.01 & 0.05 & 0.05 & 0.01 \\
\hline
\end{tabular}

6. Establish correction maps using an external standard method, $R^{2}$ can be obtained according to the standard curve to confirm the accuracy.

7. The standard SCFAs solutions (6 kinds of acetic acid, propionic acid, etc.) from high to low concentration were sequentially analyzed by GC. The signal-to-noise ratio $(\mathrm{S} / \mathrm{N})$ of the standard SCFA was obtained according to the analysis of the GC workstation software.

E. Recovery

1. Calculation of recovery rate by standard addition recovery method (Park et al., 2018).

2. Take 19 samples from the "A. Sample collection" for testing recovery.

3. Use 18 samples as parallel experiment and the other as a blank control without any treatment.

4. Six samples as a group, add $1 \mathrm{ml}$ of SCFAs standard solution of level 1 in Table 2. Select six samples as a group again and add $1 \mathrm{ml}$ of SCFAs standard solution of level 3 in Table 2. Then select 6 samples as a group and add $1 \mathrm{ml}$ of SCFAs standard solution of grade 5 in Table 2 . The purpose is to check the accuracy of recovery.

5. Treat the 19 samples in accordance with the sample pretreatment (Procedure B).

6. Analyze the processed samples according to $G C$ experimental conditions.

7. Obtain the content of SCFAs according to the standard curve of SCFAs.

F. Sample analysis

The 3 samples were analyzed according to the GC analysis method, and the SCFAs content in the samples could be obtained according to the standard curves of various SCFAs. 


\section{Data analysis}

A. GC analysis of SCFAs

1. The standard value of SCFA was determined by GC. According to the peak time obtained by $\mathrm{GC}$ for a single standard substance, the peak time of each substance of the mixed standard substance can be determined.

2. The result is shown in Figure 1 (Zhang et al., 2019). Each elute should be clearly separated and the shape of the peal sharp and symmetrical. The peak of solvent ethyl acetate is $5 \mathrm{~min}$, and the peak times of acetic acid, propionic acid, isobutyric acid, butyric acid, isovaleric acid and valeric acid were 7.886, 8.531, 8.724, 9.174, 9.472, and $9.998 \mathrm{~min}$, respectively.

3. Total GC analysis time was $13.5 \mathrm{~min}$.

B. Linearity and Sensitivity

1. LOD and LOQ were determined from the $S / N$ values of each SCFA.

2. The signal/noise $(\mathrm{S} / \mathrm{N})$ values of the standard SCFAs were 3 and 10 , respectively, which function as the limit of detection (LOD) and limit of quantification (LOQ) of each SCFA.

3. The results should be almost the same as in Table 1 (Zhang et al., 2019).

C. Recovery and accuracy

1. The results should be almost the same as in Table 2 (Zhang et al., 2019).

2. According to the peak area of SCFAs in GC analysis results and the standard curve of SCFAs, the content of SCFAs in blank samples was obtained.

3. Use the following formula to calculate the recovery rate:

$$
R(\%)=\frac{C 1-C 2}{C 3} \times 100
$$

In the equation, $\mathrm{R}$ denotes recovery, $\% ; \mathrm{C}_{1}$, is concentration of SCFAs in the standard addition and the samples; $C_{2}$, is the concentration of SCFAs in the samples; and $C_{3}$, is the concentration of SCFAs in the standard addition.

\section{$\underline{\text { Notes }}$}

1. In this experiment, young mice aged 6-8 weeks were selected. At this time, all organs of the mice were mature and the health index was good. In the following 1-2 months of the experimental cycle, the mice were healthy status.

2. In the sample collection step, after weighing the feces of mice, avoid the repeated freeze-thaw of samples. There are two benefits to storing at $-20^{\circ} \mathrm{C}$. One is to freeze the sample into clumps for easy weighing, and the other is to reduce the volatilization of the material in the feces. 
3. There are many ways to collect mice feces. This article chooses a method to deal with stimulus. The operation is as follows: fix the mouse and lift its tail, then press the mouse's lower abdomen with your fingers, collect fresh feces in a micro-centrifuge tube, store at $-20{ }^{\circ} \mathrm{C}$.

4. The number of samples is not a rigid requirement. It should be judged according to the specific experimental conditions. The purpose of selecting 12 in this article is to increase the randomness of the sample selection as much as possible to make the experimental results more accurate. If there is a clear experimental group sample, the sample size is the experimental group sample number. With regard to the amount of mice feces, it is necessary to judge at least $100 \mathrm{mg}$ for one test. If the recovery rate of six kinds of SCFAs is tested, and one SCFA is added with three different concentrations of SCFA standard solution, it will need to be tested 18 times. When the final detection of SCFAs in the sample, ensure the accuracy, test 3 times, take the average value, and include blank Group, such a system operation will need to be tested 22 times, conservatively requires $2,200 \mathrm{mg}$. In calculating the loss during weighing and other experiments, at least $3,000 \mathrm{mg}$ is collected.

5. When using $1 \mathrm{M} \mathrm{HCl}$, configure the new reagent according to the recipes. Do not use reagents configured long ago

6. When using a vortex mixer to mix $\mathrm{HCl}$ with the sample, the centrifuge tube should be tightly covered to prevent the mixture from flying out.

7. The reason for apparent lumping is that during pretreatment, the sample is frozen, and there may be caking. Another possibility is that the fecal sample itself has caking, which needs to be shaken to separate the caking and fully reflect with $\mathrm{HCl}$.

8. During ultrasonic treatment in the ice water bath, add ice in time to maintain the temperature.

9. The purpose of using the pipette gun is to operate easily and sensitively, and to control the suction at any time.

10. During the extraction operation, the centrifuge tube needs to be inverted up and down several times to fully react the supernatant with ethyl acetate. The step lasts $3 \mathrm{~min}$.

11. Pay attention to the liquid level dividing line, and do not over aspirate. The disposable syringe is not sensitive enough for operation, so special attention should be paid to it. Alternatively, use a pipette tip to transfer the supernatant to another tube and then filter to avoid disturbing the sediment or the bottom layer.

12. When using $0.22 \mu \mathrm{m}$ organic filter membrane to filter the supernatant, it is necessary to control the pressure of the needle tube to prevent the filter membrane from being broken.

13. When configuring a single standard solution or a mixed standard solution, try to configure a large-capacity solution or a high-concentration solution as much as possible to reduce the concentration error in the solution preparation.

14. When performing a gradient dilution operation, select the appropriate volumetric flask for constant volume.

15. According to the content of SCFAs in mice feces, the setting range of SCFAs concentration in single standard solution or mixed standard solution was determined. 
16. The purpose of analyzing a single standard solution is to determine the species of each substance in the mixed standard solution.

17. Because the GC injection volume is very small, when performing gradient dilution, you can choose a small volume volumetric flask to make the volume, the purpose is to reduce the waste of solution.

18. When analyzing a single standard solution, you can choose the order from low concentration to high concentration, we only need to obtain the peak time.

19. The retention time and the detection range of each analyte may vary according to capillary column type and length and equipment brands.

\section{$\underline{\text { Recipes }}$}

1. $1 \mathrm{M} \mathrm{HCl}$ solution $(100 \mathrm{ml})$

$8.35 \mathrm{ml} \mathrm{HCl} 37 \%(\mathrm{v} / \mathrm{v})$ into a $100 \mathrm{ml}$ volumetric flask

Add distilled water to constant volume to scale

2. SCFAs single standard solution (100 ml)

The highest concentrations of acetic acid, propionic acid, isobutyric acid, butyric acid, isovaleric acid, and valeric acid are $2,0.5,0.1,0.5,0.5$, and $0.1 \mathrm{~g} / \mathrm{L}$. (The label is NO.1)

$190.48 \mu \mathrm{l}$ analytical standard acetic acid, GC assay $\geq 99.8 \%$

$50.51 \mu \mathrm{l}$ analytical standard propionic acid, GC assay $\geq 99.8 \%$

$10.53 \mu \mathrm{l}$ analytical standard isobutyric acid, GC assay $\geq 99.8 \%$

$52.08 \mu \mathrm{l}$ analytical standard butyric acid, GC assay $\geq 99.8 \%$

$53.72 \mu \mathrm{l}$ analytical standard isovaleric acid, GC assay $\geq 99.8 \%$

$10.65 \mu \mathrm{l}$ analytical standard valeric acid, GC assay $\geq 99.8 \%$

Add ethyl acetate to $100 \mathrm{ml}$ volumetric flask respectively for constant volume. (Six single standard solutions are placed in six volumetric flasks respectively); Vortex for $1 \mathrm{~min}$

$$
\rho=\frac{m}{v}
$$

In the equation, $\rho$ is the density of substance, $\mathrm{g} / \mathrm{ml} ; \mathrm{m}$ is the mass of substance, $\mathrm{g} ; \mathrm{v}$ is the volume of substance, $\mathrm{ml}$

3. Dilute SCFAs single standard solution with a dilution factor of $2,4,8,10$ times

Dilute 2 times: accurately aspirate $25 \mathrm{ml}$ of No. 1 solution, add it to a $50 \mathrm{ml}$ volumetric flask, and add ethyl acetate to the mark. (The label is No.2)

Dilute 4 times: accurately aspirate $25 \mathrm{ml} \mathrm{No} .2$ solution, add it to a $50 \mathrm{ml}$ volumetric flask, and add ethyl acetate to the mark. (The label is No.3)

Dilute 8 times: accurately aspirate $25 \mathrm{ml} \mathrm{No} .3$ solution, add it to a $50 \mathrm{ml}$ volumetric flask, and add ethyl acetate to the mark. (The label is No.4)

Dilute 10 times: accurately aspirate $5 \mathrm{ml}$ of No. 1 solution, add it to a $50 \mathrm{ml}$ volumetric flask, and 
add ethyl acetate to the mark. (The label is No.5)

4. SCFAs mixed standard solution

The maximum concentration of mixed stock solution of acetic acid, propionic acid, isobutyric acid, butyric acid, isovaleric acid and valeric acid were 2, 0.5, 0.1, 0.5, 0.5 and $0.1 \mathrm{~g} / \mathrm{L}$, respectively (The labeling is No.6)

$190.48 \mu$ l analytical standard acetic acid, GC assay $\geq 99.8 \%$

$50.51 \mu \mathrm{l}$ analytical standard propionic acid, GC assay $\geq 99.8 \%$

$10.53 \mu \mathrm{l}$ analytical standard isobutyric acid, GC assay $\geq 99.8 \%$

$52.08 \mu \mathrm{l}$ analytical standard butyric acid, GC assay $\geq 99.8 \%$

$53.72 \mu \mathrm{l}$ analytical standard isovaleric acid, GC assay $\geq 99.8 \%$

$10.65 \mu \mathrm{l}$ analytical standard valeric acid, GC assay $\geq 99.8 \%$

Add ethyl acetate to a $100 \mathrm{ml}$ volumetric flask for constant volume (Six single standard solutions are put together in a volumetric flask), Vortex for $1 \mathrm{~min}$

5. Dilute SCFAs mixed standard solution with 2, 4, 8, 10 Dilution Times

Dilute 2 times: accurately aspirate $25 \mathrm{ml}$ of No. 6 solution, add it to a $50 \mathrm{ml}$ volumetric flask, and add ethyl acetate to the mark. (The label is No.7)

Dilute 4 times: accurately aspirate $25 \mathrm{ml}$ of No. 7 solution, add it to a $50 \mathrm{ml}$ volumetric flask, and add ethyl acetate to the mark. (The label is No.8)

Dilute 8 times: accurately aspirate $25 \mathrm{ml}$ of No. 8 solution, add it to a $50 \mathrm{ml}$ volumetric flask, and add ethyl acetate to the mark. (The label is No.9)

Dilute 10 times: accurately aspirate $5 \mathrm{ml}$ of No. 6 solution, add it to a $50 \mathrm{ml}$ volumetric flask, and add ethyl acetate to the mark. (The label is No.10)

\section{Acknowledgments}

This work has been supported by the Foundation (No. ZXKF20180304) of Tianjin Engineering Research Center of Microbial Metabolism and Fermentation Process Control.

\section{Competing interests}

All authors declare that there are no conflict of interests.

\section{Ethics}

All applicable international, national, and/or institutional guidelines for the care and use of animals were followed.

We use animal excrement for our experiment. Animals are not ours. They are samples provided to us by other research groups. 


\section{References}

1. Siri-Tarino, P. W., Chiu, S., Bergeron, N. and Krauss, R. M. (2015). Saturated fats versus polyunsaturated fats versus carbohydrates for cardiovascular disease prevention and treatment. Annu Rev Nutr 35: 517-543.

2. Wong, J. M., de Souza, R., Kendall, C. W., Emam, A. and Jenkins, D. J. (2006). Colonic health: fermentation and short chain fatty acids. J Clin Gastroenterol 40(3): 235-243.

3. Marette, A. and Jobin, C. (2015). SCFAs take a toll en route to metabolic syndrome. Cell Metab 22(6): 954-956.

4. Henningsson, A. M., Nyman, E. M. and Bjorck, I. M. (2001). Content of short-chain fatty acids in the hindgut of rats fed processed bean (Phaseolus vulgaris) flours varying in distribution and content of indigestible carbohydrates. Br J Nutr 86(3): 379-389.

5. Cummings, J. H. and Englyst, H. N. (1987). Fermentation in the human large intestine and the available substrates. Am J Clin Nutr 45(5 Suppl): 1243-1255.

6. Koh, A., De Vadder, F., Kovatcheva-Datchary, P. and Backhed, F. (2016). From dietary fiber to host physiology: short-chain fatty acids as key bacterial metabolites. Cell 165(6): 1332-1345.

7. Topping, D. L. and Clifton, P. M. (2001). Short-chain fatty acids and human colonic function: roles of resistant starch and nonstarch polysaccharides. Physiol Rev 81(3): 1031-1064.

8. Ruppin, H., Bar-Meir, S., Soergel, K. H., Wood, C. M. and Schmitt, M. G., Jr. (1980). Absorption of short-chain fatty acids by the colon. Gastroenterology 78(6): 1500-1507.

9. Stumpff, F. (2018). A look at the smelly side of physiology: transport of short chain fatty acids. Pflugers Arch 470(4): 571-598.

10. Hu, G. X., Chen, G. R., Xu, H., Ge, R. S. and Lin, J. (2010). Activation of the AMP activated protein kinase by short-chain fatty acids is the main mechanism underlying the beneficial effect of a high fiber diet on the metabolic syndrome. Med Hypotheses 74(1): 123-126.

11. Xiong, Y., Miyamoto, N., Shibata, K., Valasek, M. A., Motoike, T., Kedzierski, R. M. and Yanagisawa, M. (2004). Short-chain fatty acids stimulate leptin production in adipocytes through the G protein-coupled receptor GPR41. Proc Natl Acad Sci U S A 101(4): 1045-1050.

12. Johnstone, R. W. (2002). Histone-deacetylase inhibitors: novel drugs for the treatment of cancer. Nat Rev Drug Discov 1(4): 287-299.

13. O'Keefe, S. J. (2016). Diet, microorganisms and their metabolites, and colon cancer. Nat Rev Gastroenterol Hepatol 13(12): 691-706.

14. Fiorini, D., Pacetti, D., Gabbianelli, R., Gabrielli, S. and Ballini, R. (2015). A salting out system for improving the efficiency of the headspace solid-phase microextraction of short and medium chain free fatty acids. J Chromatogr A 1409: 282-287.

15. De Baere, S., Eeckhaut, V., Steppe, M., De Maesschalck, C., De Backer, P., Van Immerseel, F. and Croubels, S. (2013). Development of a HPLC-UV method for the quantitative determination of four short-chain fatty acids and lactic acid produced by intestinal bacteria during in vitro fermentation. $J$ Pharm Biomed Anal 80: 107-115. 
Please cite this article as: Zhang et. al., (2020). Gas Chromatography Detection Protocol of Short-chain Fatty Acids in Mice Feces,Bio-protocol 10 (13):

16. Torii, T., Kanemitsu, K., Wada, T., Itoh, S., Kinugawa, K. and Hagiwara, A. (2010). Measurement of short-chain fatty acids in human faeces using high-performance liquid chromatography: specimen stability. Ann Clin Biochem 47(Pt 5): 447-452.

17. de Sá, L. R. V., de Oliveira, M. A. L., Cammarota, M. C., Matos, A. and Ferreira-Leitão, V. S. (2011). Simultaneous analysis of carbohydrates and volatile fatty acids by HPLC for monitoring fermentative biohydrogen production. Int J Hydrogen Energy 36(23): 15177-15186.

18. Schiffels, J., Baumann, M. E. and Selmer, T. (2011). Facile analysis of short-chain fatty acids as 4-nitrophenyl esters in complex anaerobic fermentation samples by high performance liquid chromatography. J Chromatogr A 1218(34): 5848-5851.

19. Saavedra, L., Garcia, A. and Barbas, C. (2000). Development and validation of a capillary electrophoresis method for direct measurement of isocitric, citric, tartaric and malic acids as adulteration markers in orange juice. $J$ Chromatogr A 881(1-2): 395-401.

20. Horspool, L. J. and McKellar, Q. A. (1991). Determination of short-chain fatty acids in equine caecal liquor by ion exchange high performance liquid chromatography after solid phase extraction. Biomed Chromatogr 5(5): 202-206.

21. Destandau, E., Vial, J., Jardya, A., Henniona, M. C., Bonnet, D. and Lancelin, P. (2005). Development and validation of a reversed-phase liquid chromatography method for the quantitative determination of carboxylic acids in industrial reaction mixtures. $J$ Chromatogr $A$ 1088(1-2): 49-56.

22. Zhao, G., Nyman, M. and Jönsson, J. Å. (2006). Rapid determination of short-chain fatty acids in colonic contents and faeces of humans and rats by acidified water-extraction and direct-injection gas chromatography. Biomed Chromatogr 20(8): 674-682.

23. Hoving, L. R., Heijink, M., van Harmelen, V., van Dijk, K. W. and Giera, M. (2018). GC-MS analysis of short-chain fatty acids in feces, cecum content, and blood samples. Methods Mol Biol 1730: 247-256.

24. Zhang, C., Fan, L. and Zhao, H. (2019). Rapid Detection of Short-Chain Fatty Acids in Biological Samples. Chromatographia 83(2): 305-310.

25. Park, J., Choi, S., Oh, D. and Mah, J. H. (2018). Simultaneous and rapid analysis of chemical preservatives in processed animal products by ultra-performance liquid chromatography. Food Sci Biotechnol 27(1): 291-298. 